\title{
Further Developments of New Methods in Heat-Flow Analysis ${ }^{\dagger}$
}

\author{
M. A. BIOT* \\ Cornell Aeronautical Laboratory, Inc.
}

\section{SUMMARY}

Lagrangian methods in heat-flow problems and transport phenomena were introduced by the writer in some previous work. The present paper develops further one particular aspect of the method,-i.e., the elimination of "ignorable coordinates." This is accomplished by a special choice of generalized coordinates, each of which is constituted by an arbitrary temperature distribution and an "associated flow field." The latter is a vector field which is derived from the corresponding scalar field by a variational method. The procedure is valid for a certain class of nonlinear problems, provided we replace the temperature by the heat content as the unknown. It is shown that for normal coordinates derivation of the associated flow field is immediate. The use of normal coordinates and their associated flow fields is illustrated by an example. Introduction of Dirac functions and associated flow fields yields a procedure which constitutes a generalization of the classical formulation by Green's functions and integral equations. This is illustrated by application to onedimensional problems of heating of a homogeneous or composite slab and directly verified by classical methods in the Appendix.

\section{(1) INTRODUction}

$\mathbf{I}^{N}$ N A PREVIOUS PUBLICATION ${ }^{1}$ we have introduced new methods in heat-flow analysis. These methods have a two-fold basis; first, a new formulation of the thermodynamics of irreversible processes, and, second, the application of Lagrangian techniques to the mathematical analysis itself. The earlier developments were carricd out in references 2,3 , and 4 . We have recently reviewed in more detail in reference 5 the basic thermodynamic concepts and principles.

The formulation of dissipative phenomena into a variational language can be achieved in many ways. $\neq$ The particular method which we have chosen is different from the classical variational approaches. It appears to be the most general and fits into a unified thermodynamic theory embracing a large category of physical phenomena, which leads to equations of the same type as in Lagrangian mechanics. A particularly useful concept which has been introduced is the generalized thermodynamic force by a principle of virtual work. In the case of thermal problems this results in a representation of the physical system by means of a vectorial flow field and to the use of a generalized thermal force to represent the externally applied tem-

Received July 2. 1958.

$\dagger$ This paper is based on Cornell Aero. Lab. Report SA-987-S-5, May, 1958.

* Consultant.

‡ Other variational formulations of heat conduction have also been proposed by Rosen ${ }^{6}$ and by Chambers ${ }^{7}$ In contrast to our approach, they do not introduce the flow field and use expressions containing the temperature gradient. The relations are purely formal, and conservation of energy is not preserved. peratures. It also leads to new methods of numerical analysis of considerable advantage from the standpoint of simplicity and accuracy.

Our purpose here is to further develop one aspect of the theory which was only briefly discussed in reference 1. Heat flow may be separated into one part which represents the temperature field and another which is divergence-free and has no effect on the temperature and which we have referred to as "ignorable" in analogy with the coordinates of the same name in classical dynamics. We have also previously shown that these ignorable coordinates may be uncoupled from those representing the temperature alone, thereby reducing appreciably the number of unknown variables in the equations.

The present paper discusses and introduces additional properties of those generalized coordinates which are atitomatically uncoupled with the ignorable coordinates. In particular, this is achieved by choosing for each generalized coordinate a certain spatial temperature configuration of normalized amplitude and associating with it a certain vector field which we have referred to as the "associated flow field." The two fields are tied to each other and represent one simple generalized coordinate. In order to determine a particular coordinate we first choose a certain temperature configuration. The associated vector field is then derived from this temperature distribution by a variational method, using a principle of minimum dissipation. In a nonlinear problem we must choose a heat content configuration instead of a temperature field. Having thus determined a set of generalized coordinates, we are in a position to establish the Lagrangian differential equations which yicld the time history of the system. Attention is called to the fact that the calculation is performed in steps. The first involves the evaluation of the associated flow field, and the second the evaluation of the Lagrangian equations. Each of these steps makes use only of variational methods and do not involve any spatial differentiation of the approximate field distribution adopted as generalized coordinates. This results in improved accuracy since none of the steps involve differentiation while the integrals used in evaluating the Lagrangian equations represent an averaging process over volumes or surfaces. The procedure is in contrast with classical variational methods which require the evaluation of the temperature gradient. The separation of the calculation into two successive steps with a concurrent reduction in the number of unknowns 
greatly facilitates the numerical work without sacrifice in accuracy.

In reference 1 we have treated some one-dimensional problems by a method equivalent to the use of an associated flow field. These examples did not require the development of a formal theory because, in the particular problems treated, the flow field was already determined uniquely by the temperature distribution from the equation of conservation of thermal energy. This simplification, of course, is only applicable in certain exceptional cases and does not hold for problems in two or three dimensions. Of particular interest is the relation of the method of associated fields to the classical Green's function formulation. We will show that the latter may be derived as a very particular case of the present procedure. The introduction of associated flow fields along with a Dirac function representation of the temperature leads to integral equations which are shown to be equivalent to a Green's function method. In this formulation Green's function does not appear in its classical form but as an integral of the scalar product of two associated flow fields. By the same token we obtain a new procedure for the evaluation of Green's functions by approximate variational methods.

In Section (2) we have reviewed the fundamental equation derived in the previous work. We also indicated how these equations may be applied to the case where the thermal system contains distributed internal heat sources. This is of importance even in problems which do not involve physical sources, because they may sometimes be reduced mathematically to equivalent problems with such sources which may then be solved more easily. Section (3) discusses a general method of eliminating the ignorable coordinates by the use of a flow potential. This is carried one step further in Section (1) which develops the concept of the associated flow field as a practical method of eliminating the ignorable coordinates. Variational methods for the determination of the associated flow field directly as a vector field are discussed.

In Section (5) it is shown how the associated flow field is immediately derived without further calculation for the case of normal coordinates. An example of normal coordinates is discussed in Sections (6) and (7) for the two-dimensional problem of a flat cylinder of double wedge cross-section. It is also shown that formulation of a problem with normal coordinates requires only a knowledge of the temperature distribution of each coordinate, the corresponding eigenvalue (i.e., the relaxation constant), and the normal component of the associated flow field at the boundary. Sections (8) and (9) establish integral equations and Green's function methods as a particular case of the method of associated flow fields. Section (9) illustrates the significance of the general formulation on the particular example of the one-dimensional problem of heating of a slab across the thickness. The expression for the Green's function which follows immediately from the application of the concept of associated flow fields is verified by classical methods in the Appendix. Fi nally, in Section (10) it is shown that the method of associated flow fields may be extended to include problems where the surface heat-transfer coefficient is a function of time.

Attention is called to the general applicability of the present results to problems other than heat transfer. The methods are directly applicable to a large class of transport phenomena obeying the same type of equations of both linear and nonlinear character. In this category we may list the problems of diffusion of solutions in chemical engineering, mass diffusion of thermal neutrons in nuclear reactors, and fluid flow through porous media as encountered in problems of petroleum engineering.

\section{(2) Outline of the General Theory}

Let us first recall some of the general results which were derived in an earlier publication." We represented the thermal field by the heat flow vector field.

$$
H=H\left(q_{1} q_{2} \ldots q_{n} x y z t\right)
$$

This may be considered as a parametric vector field. The field depends on the coordinates $x, y, z$, the time $t$, and $n$ unknown parameters $q_{1} q_{2} \ldots q_{n}$. These parameters may be considered as generalized coordinates. The thermal problem consists in determining how these generalized coordinates must vary as a function of time in order to represent the actual heat flow phenomenon.

The theory was developed for the very general case of linear and nonlinear physical properties-i.e., the heat capacity, thermal conductivity, and heat-transfer coefficients may be functions of the temperatures, the coordinates, and the time.

We have shown that the equations for the unknowns $q_{i}$ are

$$
\left(\partial V / \partial q_{i}\right)+\left(\partial D / \partial \dot{q}_{i}\right)=Q_{i}
$$

They are $n$ differential equations involving the time derivatives $\dot{q}_{i}$. The function $V$ of $q_{i}$ was referred to as the thermal potential and the function $D$ of $q_{i}$ and $\dot{q}_{i}$ as the dissipation function. The vector $Q_{i}$ is the generalized thermal force.

The thermal potential is defined as the volume integral

where

$$
\begin{gathered}
V=\iiint_{\tau} F d \tau \\
F=\int_{0}^{\theta} c \theta d \theta
\end{gathered}
$$

with $c$ the heat capacity per unit volume and $\theta$ the temperature. The heat capacity may be a function of the coordinates and the temperature. The thermal potential $V$ is a function of the flow field $H$ by the relation expressing the conservation of energy-i.e.,

$$
\int_{0}^{\theta} c d \theta=-\operatorname{div} H
$$


The dissipation function is defined $\dagger$ as

$$
\begin{aligned}
& D=\frac{1}{2} \iiint_{\tau} \frac{1}{k}\left(\frac{\partial H}{\partial t}\right)^{2} d \tau+ \\
& \qquad \frac{1}{2} \iint_{S} \frac{1}{K}\left(\frac{\partial H_{n}}{\partial t}\right)^{2} d S
\end{aligned}
$$

an expression which refers to the volume $\tau$ and the heattransfer properties of the boundary $S$. The symbol $H_{n}$ refers to the normal component at the boundary taken positive outward. Since $D$ is independent of the sign of $H_{n}$ we may take it as positive either inward or outward. However for the sake of uniformity the subscript $n$ will refer to the positive outward direction. The thermal conductivity $k$ in the volume $\tau$ may be a function of the temperature and the coordinates. The surface heat-transfer coefficient is denoted by $K$ and may also be a function of the coordinates, the time, and the temperature. As shown in reference 1 , a similar expression may also be written if we wish to take care of surface radiation.

Finally, we define a generalized thermal force $Q_{i}$ by a method of virtual work. If $\theta_{a}$ denotes the external temperature applied outside of the heat-transfer layer at the surface we write

$$
Q_{i} \delta q_{i}=\iint_{S} \theta_{a} \delta H_{(n)} d S
$$

where $\delta H_{(n)}$ is the variation due to $\delta q_{i}$ alone of the normal component of the heat flow vector at the boundary $S$, its sign being positive inward. We see that expression (2.7) is equivalent to the virtual work at the boundary by the external temperature $\theta_{a}$ on the virtual heat flow $\delta H_{(n)}$. We use the subscript $(n)$ to indicate that the normal component is taken positive inward in contrast to $H_{n}$ which is positive outward as indicated above. The value of $Q_{i}$ may be written

$$
Q_{i}=\iint_{S} \theta_{a}\left[\partial H_{(n)} / \partial q_{i}\right] / d S
$$

If the surface heat transfer is through an aerodynamic boundary layer the temperature $\theta_{a}$ is the so-called adiabatic temperature of the layer.

In the particular case of a linear system-i.e., if its properties are independent of the temperature-the thermal potential becomes

$$
V=(1 / 2) \iiint c \theta^{2} d \tau
$$

and relation (2.5) is replaced by

$$
c \theta=-\operatorname{div} H
$$

In the present developments we shall be concerned primarily with systems which are linear and whose properties are also independent of time. Furthermore. we shall represent the flow field linearly in terms of the variables, $q_{i}$, by

$\uparrow \mathrm{We}$ are assuming here an isotropic material. The more general case of anisotropic media was developed in detail in reference 1 .

$$
H=\sum^{i} H_{i} q_{i}
$$

where

$$
H_{i}=H_{i}(x y z)
$$

are fixed configuration flow fields. In this case the thermal potential and dissipation functions are

$$
\left.\begin{array}{l}
V=(1 / 2) \sum_{i j}^{i j} a_{i j} q_{i} q_{j} \\
D=(1 / 2) \sum^{i j} b_{i j} \dot{q}_{i} \dot{q}_{j}
\end{array}\right\}
$$

with constant coefficients $a_{i j}$ and $b_{i j}$.

The above equations do not include the case where heat is generated inside the body. The heat conduction equation in this case becomes

$$
\operatorname{div}(k \operatorname{grad} \theta)=c(\partial \theta / \partial t)-w
$$

where $w$ is the heat produced per unit time and per unit volume. In many engineering problems such as those involving electric heating and nuclear reactors internal heat generation must be taken into account. The equations above may be used in this case provided we replace Eq. (2.5) by

$$
\int_{0}^{t} w d t-\int_{0}^{\theta} c d \theta=\operatorname{div} H
$$

This procedure is valid for both the linear and nonlinear problem. That Eqs. (2.15) and (2.2) lead to the correct formulation of the heat flow problem when there is internal heat generation may be verified by proceeding exactly as in Scctions (3) and (8) of reference 1 . In this case, a particular method of formulation of the general equations for the thermal coordinates is obtained by putting

$$
\begin{aligned}
& \int_{0}^{t} w d t=\operatorname{div} H^{*} \\
& \int_{0}^{\theta} c d \theta=-\operatorname{div} H^{+}
\end{aligned}
$$

with

$$
H=H^{+}+H^{*}
$$

The field $H^{*}$ is any field satisfying relation (2.16). Once chosen it is a given function of $x y z t$. The unknown portion of the field

$$
H^{+}=H^{+}\left(q_{1} q_{2} \ldots q_{n} x y z t\right)
$$

is a function of the unknown generalized coordinates. The temperature is defined in terms of $\boldsymbol{H}^{+}$by relation (2.17). The differential equations for $q_{i}$ are the same as Eq. (2.2) above, with a thermal potential (2.3), a dissipation function

$$
\begin{aligned}
& D=(1 / 2) \iiint_{\tau}(1 / k)\left[\left(\partial H^{+} / \partial t\right)+\left(\partial H^{*} / \partial t\right)\right]^{2} d t+ \\
& (1 / 2) \iint_{S}(1 / K)\left[\left(\partial H_{n}+/ \partial t\right)+\left(\partial H_{n}{ }^{*} / \partial t\right)\right]^{2} d S
\end{aligned}
$$

and a thermal force

$$
Q_{i}=\iint_{S} \theta_{a}\left(\partial H_{(n)}+/ \partial q_{i}\right) d S
$$


The introduction of heat sources in the problem may also result from a purely formal viewpoint in a linear physical problem in which there are no actual heat sources. This arises if we consider the heat conduction equation (2.14) with $w=0$ and put

$$
\theta=\theta^{+}+\theta^{*}
$$

where $\theta^{*}$ is the steady-state temperature distribution which would occur under the particular instantaneous external temperatures applied to the systems at any given time. This temperature satisfies the equation

$$
\operatorname{div}\left(k \operatorname{grad} \theta^{*}\right)=0
$$

and the instantaneous boundary conditions at time $t$. Since we are dealing with a linear problem, the temperature $\theta^{+}$then satisfies the equation

$$
\operatorname{div}\left(k \operatorname{grad} \theta^{+}\right)=c\left(\partial \theta^{+} / \partial t\right)+c\left(\partial \theta^{*} / \partial t\right)
$$

The field $\theta^{+}$corresponds to the case where the externally applied temperature $\theta_{a}$ is zero, with heat sources

$$
w=-c\left(\partial \theta^{*} / \partial t\right)
$$

which are given functions of time, distributed throughout the body. We therefore apply relations (2.16) and (2.17). In the present case they become

$$
\begin{aligned}
& c \theta^{*}=-\operatorname{div} H^{*} \\
& c \theta^{+}=-\operatorname{div} H^{+}
\end{aligned}
$$

where $H^{*}$ is any particular field satisfying relation (2.26). The differential equations for the field $H^{+}$are then,

$$
\left(\partial V / \partial q_{i}\right)+\left(\partial D / \partial \dot{q}_{i}\right)=0
$$

with the thermal potential

$$
V=(1 / 2) \iiint c \theta^{+2} d \tau
$$

and a dissipation function given by Eq. (2.20).

We have already discussed in reference 1 the calculation of a transient thermal field by separation into an instantaneous steady state and a remainder. A further example of such separation will also be considered hereafter [see Section (7)].

\section{(3) The Concept of Flow Potential}

We shall now discuss a particular aspect of the general method which was already briefly touched upon in reference 1 and greatly simplifies its application. The particular procedure we have in mind results from the representation of the flow field as the sum of two fields with different properties. We introduce a scalar field

$$
\psi=\psi\left(q_{1} \ldots q_{n} x y z t\right)
$$

which is a function of the generalized coordinates $q_{i}$, the space coordinates $x, y, z$, and which may or may not contain the time explicitly. We shall refer to $\psi$ as the flow potential. We write the heat flow field as

$$
H=-k \operatorname{grad} \psi+F
$$

where $F$ is a vector field

$$
\boldsymbol{F}=\boldsymbol{F}\left(f_{\mathrm{l}} \ldots f_{s}\right)
$$

which is a function of $s$ generalized coordinates $f_{s}$ and chosen such that it is divergence free,

$$
\operatorname{div} \boldsymbol{F}=0
$$

The total flow field $H$ is thus a function of $n+s$ coordinates $q_{i}$ and $f_{i}$. The temperature $\theta$ is then given by $\psi$ alone-i.e.,

$$
c \theta-\operatorname{div}(k \operatorname{grad} \psi)
$$

We shall first assume that the heat capacity $c(x, y, z)$ and the thermal conductivity $k(x, y, z)$ are either constant or functions of the coordinates but are independent of the time or the temperature. For the time being, the surface heat-transfer coefficient $K(x, y, z)$ is assumed to be either a constant or a function of the location only. The more general case is examined in Section (10). The possibility of including the case of a timeand temperature-dependent heat-transfer coefficient has an important bearing on problems of aerodynamic heating for variable velocity boundary layers. The case where $c$ and $k$ are temperature-dependent will be briefly discussed at the end of this section.

The reason for splitting up the field into two parts as in Eq. (3.2) is that for many problems we are interested in the temperature distribution only, and not in the heat flow. Now $F$, being a divergence-free field, does not contribute to the temperature. Hence, somehow the problem would be simplified if we could ignore this field and find equations which involve only $\psi$. In other words, we are interested in decoupling the two fields $\psi$ and $F$.

We will show that this can be done by choosing $\psi$ in an appropriate way. Consider first the thermal potential

$$
V=(1 / 2) \boldsymbol{\int S \int} c \theta^{2} d \tau
$$

The value of $\theta$ is determined by Eq. (3.5). Hence, the thermal potential does not contain $F$ and depends only on the coordinates $q_{i}$. This is also true if heat is generated internally, in which case we must use Eq. (2.15). Next, consider the dissipation function

$$
\begin{array}{r}
D=(1 / 2) \iiint_{\tau}(1 / k)(\partial H / \partial t)^{2} d \tau+ \\
(1 / 2) \iint_{S}(1 / K)\left(\partial H_{n} / \partial t\right)^{2} d S
\end{array}
$$

From Eq. (3.2) we derive

$$
\begin{gathered}
\partial H / \partial t=-k \operatorname{grad} \psi+(\partial F / \partial t) \\
\partial H_{n} / \partial t=-k \operatorname{grad}_{n} \psi+\left(\partial F_{n} / \partial t\right)
\end{gathered}
$$

Where $\operatorname{grad}_{n} \psi$ and $\partial F_{n} / \partial t$ are the normal components at the boundary of the vectors grad $\psi$ and $\partial F / \partial t$. $\quad \Lambda \mathrm{s}$ pointed out above, we may choose the positive direction of these normal components as we please. In conformity with our previous choice, the subscript $n$ denotes the normal component taken positive outward. Substituting expressions (3.8) and (3.9) in the dissipa- 
tion function (3.7), we may write it as the sum of three terms:

$$
D=D_{\theta}+D_{\theta f}+D_{f}
$$

where

$$
\begin{aligned}
& D_{\theta}=(1 / 2) \iiint_{\tau} k(\operatorname{grad} \psi)^{2} d \tau+ \\
& (1 / 2) \iint_{S}\left(k^{2} / K\right)\left(\operatorname{grad}_{n} \psi\right)^{2} d S \\
& D_{\theta f}=-\iiint_{\tau}(\operatorname{grad} \psi)(\partial F / \partial t) d \tau- \\
& \iint_{S}(k / K)\left(\operatorname{grad}_{n} \psi\right)\left(\partial F_{n} / \partial t\right) d S \\
& D_{S}=(1 / 2) \iiint_{\tau}(1 / k)(\partial F / \partial t)^{2} d \tau+ \\
& (1 / 2) \iint_{S}(1 / K)\left(\partial F_{n} / \partial t\right)^{2} d S
\end{aligned}
$$

The coupling terms between $q_{i}$ and $f_{z}$ are contained in $D_{\theta f}$. Let us therefore attempt to find conditions under which this term vanishes. Note that because $F$ is divergence-free--i.e., satisfies relation (3.4)-we may write

$$
\operatorname{div}[\psi \cdot(\partial F / \partial t)]=(\operatorname{grad} \psi) \cdot(\partial F / \partial t)
$$

Applying the divergence theorem (also called Green's formula), we derive

$$
\iiint_{\tau}(\operatorname{grad} \psi) \cdot(\partial F / \partial t) d \tau=\iint_{S} \psi\left(\partial F_{n} / \partial t\right) d S
$$

Substituting Eq. (3.12) in the expression of $D_{\theta r}$, we find

$$
D_{\theta f}=-\iint_{S}\left[\dot{\psi}+(k / K) \operatorname{grad}_{n} \dot{\psi}\right]\left(\partial F_{n} / \partial t\right) d S
$$

This expression will vanish if $\psi$ is chosen so that on the boundary it satisfies

$$
\dot{\psi}+(k / K) \operatorname{grad}_{n} \dot{\psi}=0
$$

If the surface heat-transfer coefficient is a function of time, this is a differential relation involving the time. If $K$ is independent of time, this condition reduces to

$$
K \psi+k \operatorname{grad}_{n} \psi=C_{P}
$$

where $C_{P}$ depends only on the location on the surface. In particular, we may put $C_{P}=0$; hence,

$$
K \psi+k \operatorname{grad}_{n} \psi=0
$$

If we choose the flow potential $\psi$ to satisfy these boundary conditions, then $V$ and $D_{\theta}$ depend only on the $n$ coordinates $q_{i}$ and we may write the $n$ equations

$$
\left(\partial V / \partial q_{i}\right)+\left(\partial D_{\theta} / \partial \dot{q}_{i}\right)=Q_{i}
$$

with the thermal forces

$$
Q_{i}=\iint_{S} \theta_{a} k\left(\partial / \partial q_{i}\right)\left(\operatorname{grad}_{n} \psi\right) d S
$$

The other equations for the coordinates $f_{i}$,

$$
\partial D_{j} / \partial \dot{f}_{i}=0
$$

do not enter into the picture as far as the temperature field is concerned, and are relevant only if we wish to determine the amount of heat flow. As pointed out in reference 1 , the coordinates may be called "ignorable coordinates" in analogy with the similar case in dynamics when such coordinates appear only in the expression for the kinetic energy. For instance, in a free elastic solid, the ignorable coordinates are those corresponding to rigid translations and rotations. They are irrelevant if we are interested only in the strains.

If the thermal field contains surfaces of discontinuity for the conductivity $k$ we may still use a flow potential $\psi$, which will have the same properties as above provided both $\psi$ and the component of $k \operatorname{grad} \psi$ normal to the surface of discontinuity be continuous across this surface. Since $\psi$ is continuous along the surface of discontinuity this implies a refraction of the flow lines across the surface.

We have assumed until now that $c, k$, are independent of the temperature. If $c$ is temperaturc-dependent, the method is applicable, except that Eq. (3.5) must be replaced by

$$
h(\theta)=\int_{0}^{\theta} c d \theta=\operatorname{div}(k \operatorname{grad} \psi)
$$

If $k$ is also temperature-dependent, but independent of the coordinates, it is well known that the problem may be reduced to one with a constant value for $k$ (see reference 1). Finally, the method may be extended to the case of anisotropic media for the case where the conductivity is temperature-independent.

\section{(4) Temperature Fields and Their Assochated Flow Fields as Generalized Coordinates}

In the particular case of a system with physical properties independent of the temperature and the time, the quantities $c, k$, and $K$ are functions only of the coordinates. The flow potential may then conveniently be represented by

$$
\psi=\sum^{i} \psi_{i}(x y z) q_{i}
$$

The corresponding temperature field is

$$
\theta=\sum^{i} \theta_{i}(x y z) q_{i}
$$

and the corresponding flow field is

$$
H=\sum^{i} \boldsymbol{\theta}_{i}(x y z) q_{i}
$$

with

$$
\begin{gathered}
\theta_{i}=(1 / c) \operatorname{div}\left(k \operatorname{grad} \psi_{i}\right) \\
\boldsymbol{\Theta}_{i}=-k \operatorname{grad} \psi_{i}
\end{gathered}
$$

In addition, for the field to be uncoupled with the ignorable coordinates each function $\psi_{i}$ must satisfy the boundary condition

$$
K \psi_{i}+k \operatorname{grad}_{n} \psi_{i}=0
$$


This result was already established in reference 1 and is also a consequence of the more general relations (3.14) derived above.

The point of importance which we shall now discuss is the fact that Eq. (4.4) and the boundary condition (4.6) completely define the flow potential $\psi_{i}$ when $\theta_{i}$ is given. The function $\psi_{i}$ is simply the steady-state temperature for heat sources of intensity $-c \theta_{i}$ per unit volume and per unit time distributed throughout the system while the external temperature is maintained zero. The field $\boldsymbol{\Theta}_{i}$ is also completely determined by $\theta_{i}$. It is the steady rate of heat flow established in this system of sources. We shall call $\Theta_{i}$ the associated flow field for the fixed temperature configuration $\theta_{i}$. Note that because of the minus sign the sources $-c \theta_{i}$ may be considered as heat sink of magnitude $c \theta_{i}$. .

In practice, it will generally be much easier to choose a number of temperature configurations $\theta_{i}$ as approximations to the problem rather than choosing suitable approximations for $\psi_{i}$. The thermal problem can then be formulated completely if we first calculate the flow fields $\boldsymbol{\Theta}_{i}$ associated with each temperature configuration. Fach scalar field $\theta_{i}$ and its associated vector field $\boldsymbol{\Theta}_{i}$ defines a generalized coordinate $q_{i}$. Now the calculation of the associated flow field may be accomplished by approximate methods, and, particularly, by variational methods. Since $\psi_{i}$ represents the steady-state temperature due to the sources, there are numerous approximate methods available for its calculation. In particular, we may use a variational method by putting equal to zero the variation of

$$
\begin{array}{r}
I=\iiint_{\tau}\left[(1 / 2) k\left(\operatorname{grad} \psi_{i}\right)^{2}+c \theta_{i} \psi_{i}\right] d \tau+ \\
(1 / 2) \iint_{S} K \psi_{i}{ }^{2} d S
\end{array}
$$

where $\psi_{i}$ is the quantity to be varied and $\theta_{i}$ is given. This leads to Eq. (4.4) with the boundary condition (4.6).

Actually, of course, we are not interested in the scalar $\psi_{i}$ but in the associated field $\Theta_{i}$, as given by relation (4.5). Since this involves the calculation of the gradient of $\psi_{i}$ it is more accurate to use the variational method, to calculate the vector field $\boldsymbol{\Theta}_{i}$ directly. This can be done by minimizing the dissipation function

$$
\begin{aligned}
D^{\prime}=(1 / 2) \iiint_{\tau}(1 / k) \Theta_{i}{ }^{2} d \tau+ \\
(1 / 2) \iint_{S}(1 / K)\left[\Theta_{i}\right]_{n}{ }^{2} d S
\end{aligned}
$$

for variations of $\Theta_{i}$ which satisfy the constraint

$$
\operatorname{div} \Theta_{i}=-c \theta_{i}
$$

This may be derived by adapting to the particular case of steady sources the considerations of Section (2) above, and the general variational principles as formulated in references 1 and 2 . The steady-state source is $w=-c \theta_{i}$ and the rate of heat flow is $\partial H / \partial t=\Theta_{i}$.

$\dagger$ The subscript $n$ indicates the component of $\boldsymbol{\Theta}_{i}$ normal to the surface $S$ and taken positive outward.
This procedure of minimizing expression (4.8) for $D^{\prime}$ under the constraint (4.9) may also be derived dircetly by introducing a Lagrangian multiplier $\lambda$. It is then equivalent to putting equal to zero the variations of

$$
\begin{array}{r}
D^{\prime \prime}=\iiint_{\tau}\left[(1 / 2 k) \boldsymbol{\Theta}_{i}{ }^{2}+\lambda\left(\operatorname{div} \boldsymbol{\Theta}_{i}+c \theta_{i}\right)\right] d \tau+ \\
(1 / 2) \int \boldsymbol{\int}(1 / K)\left[\boldsymbol{\Theta}_{i}\right]_{n}{ }^{2} d S
\end{array}
$$

We derive Euler's equations corresponding to this variational problem. These equations are, in the volume $\tau$,

$$
(1 / k) \Theta_{i}-\operatorname{grad} \lambda=0
$$

and at the boundary,

$$
\lambda+(1 / K)\left[\Theta_{i}\right]_{n}=0
$$

Taking into account the constraint (4.9), these equations lead to

$$
\begin{aligned}
& \operatorname{div}[k \operatorname{grad} \lambda]=-c \theta_{i} \\
& \lambda+(k / K) \operatorname{grad}_{n} \lambda=0
\end{aligned}
$$

Eqs. (4.11), (4.13), and (4.14) are identical with those defining $\boldsymbol{\Theta}_{i}$ in terms of $\theta_{i}$. The Lagrangian multiplier $\lambda$ plays the role of $-\psi_{i}$.

An alternate procedure which may generally be more convenient is to put

$$
\Theta_{i}=F_{i}+\Theta_{i}{ }^{\prime}
$$

where $\Theta_{i}{ }^{\prime}$ is any field satisfying the relation

$$
\operatorname{div} \boldsymbol{\Theta}_{i}{ }^{\prime}=-c \theta_{i}
$$

The field $F_{i}$ is the variable to be varied under the condition

$$
\operatorname{div} F_{i}=0
$$

The variation of

$$
\begin{aligned}
D=(1 / 2) \iiint_{\tau}(1 / k)\left(\boldsymbol{F}_{i}+\boldsymbol{\Theta}_{i}{ }^{\prime}\right)^{2} d \tau+ \\
(1 / 2) \iint_{S}(1 / K)\left[\boldsymbol{F}_{i}+\boldsymbol{\Theta}_{i}^{\prime}\right]_{n}{ }^{2} d S
\end{aligned}
$$

is then made to vanish under the constraint (4.17).

It is important to note that, in practice, we do not require the calculation of the scalar field $\psi_{i}$. Once the thermal coordinate $\theta_{i}$ has been chosen, its associated vector field $\Theta_{i}$ is determined directly, and is the only additional field required to define the generalized coordinate.

In Section (2) we have considered the case where there are internal heat sources. It was found that we could scparate the flow ficld into two parts $H^{*}$ and $\mathrm{H}^{+}$satisfying relations (2.16) and (2.17). These relations for a linear problem become

$$
\begin{gathered}
\int_{0}^{t} w d t=\operatorname{div} H^{*} \\
c \theta=-\operatorname{div} H^{+}
\end{gathered}
$$

The field $H^{*}$ is any field satisfying the first equation. 
The concept of the associated flow field may also be used in this case by considering as above the representations

$$
\begin{aligned}
\theta & =\sum \theta_{i} q_{i} \\
H^{+} & =\sum \boldsymbol{\Theta}_{i}{ }^{+} q_{i}
\end{aligned}
$$

The field $\Theta_{i}{ }^{+}$is associated with the temperature $\theta_{i}$ by the relations

$$
\begin{gathered}
\theta_{i}=(1 / c) \operatorname{div}\left(k \operatorname{grad} \psi_{i}{ }^{+}\right) \\
\Theta_{i}{ }^{+}=-k \operatorname{grad} \psi_{i}{ }^{+}
\end{gathered}
$$

and the boundary condition

$$
K \psi_{i}{ }^{+}+k \operatorname{grad}_{n} \psi_{i}^{+}=0
$$

The dissipation function is expressed in terms of a total flow field

$$
H=H^{*}+H^{+}+F
$$

where $F$ satisfies the relation

$$
\operatorname{div} F=0
$$

We may consider that $F$ contains the arbitrary portion of the divergence-free field which may be added to $\bar{H}^{*}$. Repeating the argument of the previous sections and taking into account Eqs. (4.24) and (4.25) we see that in the dissipation functions the fields $F$ and $\bar{H}^{+}$are uncoupled. Hence, if we are interested only in the temperature $\theta$, we may use for the dissipation function

$$
\begin{gathered}
D=(1 / 2) \iiint_{\tau}(1 / k)\left[\left(\partial H^{*} / \partial b\right)+\left(\partial H^{+} / \partial t\right)\right]^{2} d \tau+ \\
(1 / 2) \iint_{S}(1 / K)\left[\left(\partial H^{*} / \partial t\right)+\left(\partial H^{+} / \partial t\right)\right]^{2} d S
\end{gathered}
$$

For the same reason as just mentioned, addition of a divergence-free field to $H^{*}$ introduces no new coupling between $H^{*}$ and $H^{+}$. We need of course write only those terms which include $q_{i}$; hence, we put

$$
\begin{aligned}
D=(1 / 2) \iiint_{\tau}(1 / k)\left(\sum \Theta_{i}+\dot{q}_{i}\right)^{2} d \tau+ \\
(1 / 2) \iint_{S}(1 / K)\left[\sum^{i}\left(\Theta_{i}{ }^{2}\right)_{n} \dot{q}_{i}\right]^{2} d S+ \\
\iiint_{\tau}(1 / k)\left(\partial H^{*} / \partial t\right)\left(\sum^{i} \Theta_{i}{ }^{+} \dot{q}_{i}\right) d \tau+ \\
\iint_{S}(1 / K)\left(\partial H_{n}+/ \partial t\right) \sum^{i}\left[\Theta_{i}{ }^{+}\right]_{n} \dot{q}_{i} d S
\end{aligned}
$$

The same procedure will be used when the temperature field is separated into an instantaneo:1s steady state $\theta^{*}$ and a remainder $\theta^{+}$. As pointed out above, this amounts to distributing sources (2.25) throughout. Eqs. (4.19) and (4.20) are simply replaced by Eqs. (2.26) and (2.27). The remainder temperature $\theta^{+}$is then expanded into a sum of temperature configurations $\theta_{i}+$ with associated fields

$$
\theta^{+}=\sum^{i} \theta_{i}{ }^{+} q_{i}, \quad H^{+}=\sum^{i} \Theta_{i}{ }^{+} q_{i}
$$

The fields $\boldsymbol{M}_{i}+$ associated with each temperature configuration may be evaluated by a variational method as above. Hence, the equations for $q_{i}$ for the field $\theta^{+}$are then obtained from Eq. (2.28) using the dissipation function (4.29).

\section{(5) The Associated Flow Field for Normal CoORdinates}

In many applications it is possible to determine readily the temperature fields corresponding to the thermal modes or normal coordinates. These thermal modes are decaying temperature distributions under boundary conditions of zero external temperature $\left(\theta_{a}=0\right)$. Systems where this concept applies are those for which the physical parameters $c, k$, and $K$ depend only on the coordinates. The system may or may not include a surface layer for heat transfer.

These thermal modes may be used as normal coordinates to solve the problem of finding the temperature distribution when arbitrary external temperatures $\theta_{a}$ are applied at the surface. However, in order to do this we must calculate the corresponding thermal forces by a method of virtual work, and this implies the knowledge of the heat flow field associated with each thermal mode.

We will show that the flow field associated with each mode is immediately determined from the temperature distribution. Let us represent the flow field of each thermal mode by $\Theta_{s}$ and its temperature field by $\theta_{s}$. Since we are interested only in those modes associated with a temperature field, we introduce a flow potential $\psi_{s}$ associated with $\boldsymbol{\Theta}_{s}$ and $\boldsymbol{\theta}_{s}$. From Eqs. (4.4) and (4.5) we derive

$$
\begin{gathered}
\Theta_{s}=-k \operatorname{grad} \psi_{s} \\
\operatorname{div}\left(k \operatorname{grad} \psi_{s}\right)=c \theta_{s}
\end{gathered}
$$

The boundary condition for $\psi_{s}$ is given by Eq. (4.6) i.e.,

$$
K \psi_{s}+k \operatorname{grad}_{n} \psi_{s}=0
$$

Eq. (5.2) together with Eq. (5.3) completely determines $\psi_{s}$ when $\theta_{s}$ is given. In order to find $\psi_{s}$ let us consider the temperature field. It satisfies the equation

$$
\operatorname{div}(k \operatorname{grad} \theta)=c(\partial \theta / \partial t)
$$

with the boundary condition

$$
K \theta+k \operatorname{grad}_{n} \theta=0
$$

Note that we are dealing here with a relaxation modei.e., a case where the temperature $\theta_{a}$ outside the surface layer is zero and the rate of heat transfer at the surface is measured by $K \theta$. A thermal mode is a solution of the type

$$
\theta=\theta_{s}(x y z) e^{-\lambda_{s} t}
$$

where $\theta_{s}$ is a fixed temperature field normalized in an arbitrary way. Eq. (5.4) and the boundary condition (5.5) become 

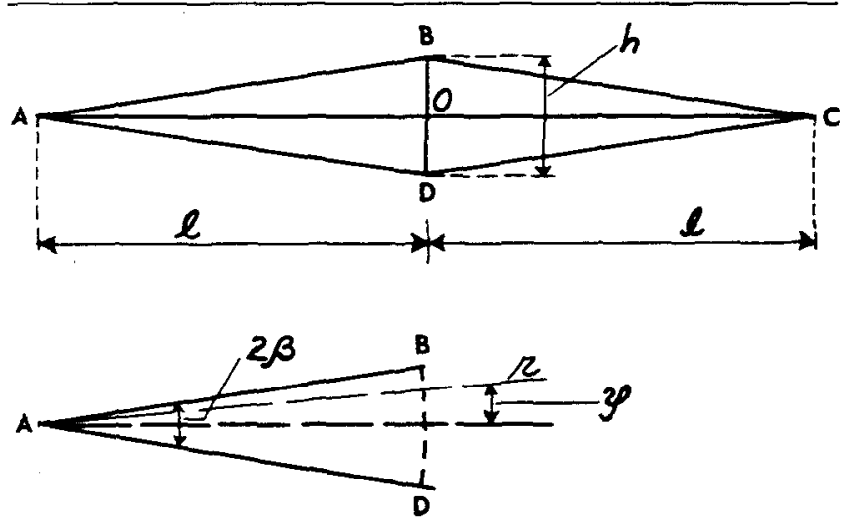

FIG. 1. Double wedge section of cylinder.

$$
\begin{gathered}
\operatorname{div}\left(k \operatorname{grad} \theta_{s}\right)=-\lambda_{s} c \theta_{s} \\
K \theta_{s}+k \operatorname{grad}_{n} \theta_{s}=0
\end{gathered}
$$

By putting

$$
\psi_{s}=-\left(1 / \lambda_{s}\right) \theta_{s}
$$

these equations may be written

$$
\begin{aligned}
& \operatorname{div}\left(k \operatorname{grad} \psi_{s}\right)=c \theta_{s} \\
& K \psi_{s}+k \operatorname{grad}_{n} \psi_{s}=0
\end{aligned}
$$

Hence, $\psi_{s}$ as given by Eq. (5.10) is the unique solution of Eq. (5.2). The significance of this result lies in the fact that for every temperature field $\theta_{s}$ corresponding to a thermal mode, there exists a vector field

$$
\boldsymbol{\Theta}_{s}=\left(k / \lambda_{s}\right) \operatorname{grad} \theta_{s}
$$

which represents the associated flow field. The scalar field $\theta_{s}$ and the associated vector field $\Theta_{s}$ constitute a complete representation of the normal coordinate which corresponds to the eigenvalue $\lambda_{s}$. This concept and its practical usefulness are illustrated by the examples in Sections (6) and (7).

\section{(6) Example of Normal Coordinates}

Let us consider a flat cylindrical body of double wedge section $A B C D$ (see Fig. 1). It is assumed that the body is homogeneous with constant heat capacity and thermal conductivity. The width of the body is $2 l$ and the maximum thickness $h$. We shall evaluate the thermal modes in the cross section considered as a two-dimensional problem. The example will be restricted to the case of modes symmetrical with respect to $\mathrm{AC}$ and $\mathrm{BD}$ and to the case where there is no boundary layer-i.e., where the heat-transfer coefficient at the surface is infinite $(K=\infty)$. The thermal modes then occur under conditions of constant temperature $(\theta=0)$ at the boundary of the solid. $\dagger$ We furthermore assume the thickness ratio $h / l$ to be small so that the half portion $\mathrm{ABD}$ may be assimilated to a section of vertex angle,

$$
2 \beta=h / l
$$

I It is clear that this assumption is not essential and that the use of normal coordinates applies quite generally to cases with a finite surface heat-transfer coefficient.
The temperature in the sector satisfies the heat conduction equation in polar coordinates $r$ and $\varphi$ with $\mathrm{A}$ as origin.

$$
\begin{aligned}
& \left(\partial^{2} \theta / \partial r^{2}\right)+(1 / r)(\partial \theta / \partial r)+ \\
& \left(1 / r^{2}\right)\left(\partial^{2} \theta / \partial \varphi^{2}\right)=(1 / \kappa)(\partial \theta / \partial t)
\end{aligned}
$$

The diffusivity is

$$
\kappa=k / c
$$

The boundary conditions for $\theta$ are

$$
\left.\begin{array}{ll}
\theta=0 & \text { for } \quad \varphi= \pm \beta \\
\partial \theta / \partial r=0 & \text { for } \quad r=l
\end{array}\right\}
$$

The arc of circle $\mathrm{BD}$ is assimilated to a straight line because $\beta$ is assumed to be small. The thermal modes are solutions of the type

$$
\theta=f(r) \cos (\nu \varphi) e^{-\lambda_{s} t}
$$

Substitution in Eq. (5.4) yields

$$
\begin{aligned}
\left(d^{2} f / d r^{2}\right)+(1 / r)(d f / d r)+ & + \\
& {\left[\left(\lambda_{s} / \kappa\right)-\left(\nu^{2} / r^{2}\right)\right] f=0 }
\end{aligned}
$$

Solutions of this equation which remain finite are

$$
f=J_{\nu}\left(r \sqrt{\lambda_{s} / \kappa}\right)
$$

This may be written

$$
\begin{aligned}
& f=J_{\nu}\left[\alpha_{s}(r / l)\right] \\
\text { with } \quad \alpha_{s} & =l \sqrt{\lambda_{s} / \kappa}
\end{aligned}
$$

The first boundary condition (6.4) is satisfied if we put

$$
\nu=(\pi / \beta)[m+(1 / 2)] \quad(m=0,1,2 \ldots)
$$

and the second boundary condition is

$$
J_{\nu}{ }^{\prime}\left(\alpha_{s}\right)=0
$$

with

$$
J_{\nu}{ }^{\prime}(x)=d J_{\nu}(x) / d x
$$

Eq. (6.11) has an infinite number of roots $\alpha_{8}$ which may be evaluated numerically by interpolation from tables of Bessel functions. We may write $\lambda_{s}$ in terms of $\alpha_{s}$

$$
\lambda_{s}=\kappa\left(\alpha_{s}^{2} / l^{2}\right)
$$

The thermal modes are therefore

$$
\theta=J_{\nu}\left[\alpha_{s}(r / l)\right] \cos (\nu \varphi) e^{-\lambda_{s} t}
$$

There is a double infinity of modes attached to the roots $\alpha$ so that the subscript $s$ has the significance of a double index. We write the thermal modes as

$$
\theta=\theta_{s} e^{-\lambda_{s} t}
$$

with

$$
\theta_{\mathrm{S}}=J_{\nu}\left[\alpha_{s}(r / l)\right] \cos \nu \varphi
$$

Any thermal distribution in the sector may be represented by a superposition of thermal modes

$$
\theta=\sum^{s} \theta_{s} q_{s}
$$

where $q_{s}$ are generalized coordinates. As we have shown in the previous section the corresponding expansion for the heat flow field is 


$$
H=\sum^{s} \boldsymbol{\omega}_{s} q_{s}
$$

where $\Theta_{s}$ is the flow field associated with the temperature distribution $\theta_{s}$. It is given by

$$
\boldsymbol{\Theta}_{s}=\left(k / \lambda_{s}\right) \operatorname{grad} \theta_{s}
$$

The thermal potential and the dissipation function are readily expressed in terms of the normal coordinates. In reference 1 we have formulated the orthogonality properties of the thermal modes. Because of these properties all cross products vanish in the expressions for $V$ and $D$. The thermal potential is

$$
V=(1 / 2) \sum^{s} a_{s} q_{s}{ }^{2}
$$

with

$$
a_{s}=(1 / 2) c \iint \theta_{s}^{2} d x d y
$$

The surface integral is extended to the area $\triangle B C$ of the sector. Similarly, the dissipation function is written

$$
D=(1 / 2) \sum^{s} b_{s} q_{s}{ }^{2}
$$

with

$$
b_{s}=(1 / 2)\left(k / \lambda_{s}{ }^{2}\right) \iint\left(\operatorname{grad} \theta_{s}\right)^{2} d x d y
$$

However, this integral need not be evaluated if we know $a_{s}$ and $\lambda_{s}$. This can be shown readily by writing the differential equations satisfied by the thermal modes in terms of the normal coordinates $q_{s}$. Since the boundary temperature is zero the thermal force $Q_{s}$ vanishes, and the general differential equation (2.2) becomes

$$
\begin{gathered}
\left(\partial V / \partial q_{s}\right)+\left(\partial D / \partial \dot{q}_{s}\right)=0 \\
a_{s} q_{s}+b_{s} \dot{q}_{s}=0
\end{gathered}
$$

The solutions of these equations are the thermal modes,

$$
q_{s}=C_{s} e^{-\lambda_{s} t}
$$

with

$$
\begin{aligned}
& \lambda_{s}=a_{s} / b_{s} \\
& b_{s}=a_{s} / \lambda_{s}
\end{aligned}
$$

The dissipation function may therefore be written

$$
D=(1 / 2) \sum^{s}\left(a_{s} / \lambda_{s}\right) \dot{q}_{s}^{2}
$$

with $a_{s}$ equal to the integral (6.20).

\section{(7) Application of Normal Coordinates}

With a knowledge of the normal coordinates we may immediately express the temperature field caused by an arbitrary time-dependent distribution of the external temperature, $\theta_{a}$, at the surface. We shall again consider the example of the previous section with infinite surface heat-transfer coefficient $(K=\infty)$ and the same symmetric distribution of temperature with respect to $\mathrm{AC}$. In addition, we shall also assume that the external temperature distribution is also symmetric with respect to $\mathrm{BD}$. Because of the assumed symmetry the temperature field need be evaluated only in the sector $\mathrm{ABO}$. Since $K=\infty$, the external temperature $\theta_{a}$ is identical with the temperature at the solid boundary.

The temperature is represented in terms of normal coordinates by the series (6.16). With each normal coordinate is associated a flow field $\Theta_{s}$ given by Eq. (3.18). The thermal potential $V$ and dissipation function $D$ are expressions (6.19) and (6.28) with coefficients $a_{s}$ defined by Eq. (6.20). The differential equations for the normal coordinates $q_{s}$ are

$$
a_{s} q_{s}+\left(a_{s} / \lambda_{s}\right) \dot{q}_{s}=Q_{s}
$$

where $Q_{s}$ is the generalized force associated with $q_{s}$. It is defined in terms of the normal component $\left[\Theta_{s}\right]_{(n)}$ of the associated flow field at the boundary by applying Eq. (2.8). We find $\dagger$

$$
Q_{s}=\iint_{S} \theta_{a}\left[\Theta_{s}\right]_{(n)} d S
$$

In the present example, since we consider only the sector $\mathrm{ABO}$, the thermal force is

$$
Q_{s}=-\left(k / \lambda_{s}\right) \int_{0}^{l}\left(\theta_{a} / r\right)\left(\partial \theta_{s} / \partial \varphi\right)_{\varphi=\beta} d r
$$

or

$$
Q_{s}=(-1)^{m}\left(k_{\nu} / \lambda_{s}\right) \int_{0}^{l}\left(\theta_{a} / r\right) J_{\nu}\left[\boldsymbol{\alpha}_{s}(r / l)\right] d r
$$

The coefficients $a_{s}$ defined by Eq. (6.20) are

$$
\begin{aligned}
a_{s}= & (1 / 2 c) \int_{0}^{l} d r \int_{0}^{\beta} r J_{\nu}{ }^{2}\left[\alpha_{s}(r / l)\right] \cos ^{2} \nu \varphi d \varphi \\
& a_{s}=\left(\beta l^{2} / 8 c\right)\left[1-\left(\nu^{2} / \alpha_{s}{ }^{2}\right)\right] J_{\nu}{ }^{2}\left(\alpha_{s}\right)
\end{aligned}
$$

The differential equations (7.1) determine $q_{s}$ as a function of time. These values of $q_{s}$ substituted in the series (6.16) determine the transient temperature field. It will be noticed that the temperature field as represented by the series is always zero right at the boundary, and therefore cannot represent the imposed boundary temperature. Nevertheless, it does represent correctly the temperature distribution inside the boundary. If, for instance, we impose a constant temperature $\theta_{a}$ on this boundary, the field will be represented by a series which has a constant value inside the boundary but whose value drops to zero if we are close enough to the boundary. This is a feature of nonuniform convergence already encountered in onedimensional examples in references 1 and 4 . There are advantages and disadvantages in expressing the temperature field by a series such as (6.16). The advantage is, of course, the orthogonality that results in the absence of coupling between the coordinates $q_{s}$. A disadvantage is the fact that the final distribution of temperature under equilibrium conditions is represented by a series. As was pointed out in reference 1 , one way of avoiding this disadvantage is to split up the temperature field into two parts. Let us apply this method to the present example. Consider the solutions of the equations

† The subscript $(n)$ denotes the normal component taken positive inward.

$\ddagger$ This result may be obtained by making use of integral expressions originally derived by E. C. J. Lommel (see reference 8). 


$$
a_{s} q_{s}^{*}=Q_{s}
$$

The $q_{s}{ }^{*}$ represent the equilibrium temperature distribution reached under instantaneous boundary temperatures $\theta_{a}$ considered as independent of time. It represents a temperature field

$$
\theta^{*}=\sum^{s} \theta_{s} q_{s}^{*}
$$

which may be evaluated directly by solving Laplace's equation

$$
\left(\partial^{2} \theta^{*} / \partial x^{2}\right)+\left(\partial^{2} \theta^{*} / \partial y^{2}\right)=0
$$

under the instantaneous boundary condition $\theta_{a}$ for the temperature. We then put

$$
\begin{gathered}
\theta=\theta^{+}+\theta^{*} \\
\theta^{+}=\sum^{s} \theta_{s} q_{s}{ }^{+} \\
q_{s}=q_{s}^{+}+q_{s}^{*}
\end{gathered}
$$

Substituting in Eqs. (7.1) and taking into account relation (7.5) gives the differential equation for $q_{s}{ }^{+}$

$$
q_{s}++\left(1 / \lambda_{s}\right) \dot{q}_{s}{ }^{+}=-\left(1 / \lambda_{s}\right) \dot{q}_{s}{ }^{*}
$$

Now from Eq. (7.5) we may also write

$$
-\left(1 / \lambda_{s}\right) \dot{q}_{s}^{*}=-\left(\dot{Q}_{s} / \lambda_{s} a_{s}\right)
$$

The time derivative $\dot{Q}_{s}$ is found by calculating the initial work of the time derivative $\dot{\theta}_{a}$ of the boundary temperature.

\section{(8) Integral Equations Derived From Associated} Flow Frelds-Relation to Green's Function

In Section (4) we have shown how temperature fields and their associated flow fields may be used as generalized coordinates in the formulations of heat flow problems. We shall now examine a particular case of this type of formulation which leads to integral equations in complete generality for all linear thermal systems with time-independent parameters. This formulation amounts to a generalization of the Green's function method and at the same time yields a new procedure for the approximate evaluation of this generalized Green's function itself.

We go back to Eq. (4.2), which represents the thermal field as

$$
\theta=\sum^{i} \theta_{i}(x y z) q_{i}
$$

where $\theta_{i}$ are fixed scalar distributions and $q_{i}$ generalized coordinates. An interesting application of this representation arises if we write Eq. (8.1) in the form

$$
\theta=\int \delta(\xi-x) \theta(x) d x
$$

where $\delta(\xi-x)$ plays the role of the configuration $\theta_{i}$, $\theta(x) d x$ represents the generalized coordinates $q_{i}$, and the summation is replaced by an integral. We have abbreviated the notation by writing $\theta(x)$ for $\theta(x y z)$. By a similar abbreviation the integral is a volume integral over $x y z$. The distribution $\delta(\xi-x)$ is written for $\delta(\xi-x, n-y, \zeta-z)$ and represents the spatial Dirac function equal to zero at any point except $\xi=x$, $\eta=y, \zeta=z$, and such that its volume integral over $\xi \eta \zeta$ is unity. By the property of Dirac functions, Eq. (8.2) is equivalent to stating that $\theta=\theta(\xi)$; i.e., the continuous set of unknown local temperatures constitute the generalized coordinates of the problem.

The flow field $\Theta(\xi, x)$ associated with the temperature $\delta(\xi-x)$ is determined by the general procedure outlined above in Section (4). We solve the equation

$$
\operatorname{div}(k \operatorname{grad} \psi)=c \delta(\xi-x)
$$

where

$$
\psi=\psi(\xi, x)
$$

with the boundary condition

$$
K \psi+k \operatorname{grad}_{n} \psi=0
$$

The gradients and all partial derivations are taken with respect to $\xi \eta \zeta$. The right-hand side of Eq. (8.3) represents a concentrated heat sink of magnitude $c$ at point $x y z$ and $\psi$ is the corresponding temperature field. The flow field associated with $\delta(\xi-x)$ is

$$
\Theta(\xi, x)=-k \operatorname{grad} \psi(\xi, x)
$$

We have also indicated in Section (5) how $\Theta$ could be evalualed by an approximate variational procedure. Eq. (4.3) for the total flow field becomes the volume integral

$$
H=\boldsymbol{\int} \boldsymbol{\Theta}(\xi, x) \theta(x) d x
$$

In order to obtain the general equations for the thermal field we must construct the thermal potential $V$ and the dissipation function $D$. The first is written immediately as

$$
V=(1 / 2) \int c \theta^{2} d x
$$

The second is found by writing first

$$
(\partial H / \partial t)^{2}=\int \boldsymbol{S} \boldsymbol{\Theta}\left(\xi, x^{\prime}\right) \cdot \boldsymbol{\Theta}(\xi, x) \dot{\theta}\left(x^{\prime}\right) \dot{\theta}(x) d x^{\prime} d x
$$

We easily derive

$$
D=(1 / 2) \boldsymbol{\int} \gamma\left(x^{\prime}, x\right) \dot{\theta}\left(x^{\prime}\right) \dot{\theta}(x) d x^{\prime} d x
$$

with

$$
\begin{array}{r}
\gamma\left(x^{\prime}, x\right)=\boldsymbol{\int}[1 / k(\xi)] \Theta\left(\xi, x^{\prime}\right) \Theta(\xi, x) d \xi+ \\
\int(1 / K) \Theta_{n}\left(\xi, x^{\prime}\right) \Theta_{n}(\xi, x) d S
\end{array}
$$

The second integral is a surface integral over the boundary where $\Theta_{n}(\xi, x)$ represents the normal component of $\Theta(\xi, x)$ at a point $\xi$ of the boundary. Finally the generalized force $Q(x)$ is obtained by considering the virtual work of the applied external temperature $\theta_{a}$ on the heat flow $\delta H_{(n)}$ at the boundary for a variation $\delta \theta(x)$. We find

$$
\begin{aligned}
Q(x) \delta \theta(x)=\int_{S} \theta_{a} \delta H_{(n)} d S & = \\
& \int_{S} \theta_{a} \Theta_{(n)}(\xi, x) \delta \theta(x) d S
\end{aligned}
$$

The normal component $\Theta_{(n)}$ is taken positive inward. We derive 


$$
Q(x, t)=\int_{S} \theta_{a}(\xi, t) \theta_{(n)}(\xi, x) d S
$$

We have written $Q(x, t)$ and $\theta_{a}(\xi, t)$ instead of $Q(x)$ and $\theta_{a}$ to indicate explicitly that these quantities are functions of both the coordinates and the time.

The final equations for the temperature field are obtained by using the concept of partial derivative with respect to $\theta(x) d x$ and $\dot{\theta}(x) d x$ as if they were coordinates $q_{i} \dagger^{\dagger}$ We find

$$
c \theta(x, t)+\int \gamma\left(x^{\prime}, x\right) \dot{\theta}\left(x^{\prime}, t\right) d x^{\prime}=Q(x, t)
$$

Again we write $\theta(x, t)$ instead of $\theta(x)$ to indicate explicitly that it is also an unknown function of time. This is an integral equation for the unknown temperature $\theta(x, t)$.

The particular form of this equation recalls immediately that obtained by the classical Green's function method. ${ }^{10}$ In fact, it is shown in the Appendix that this equation in the case of well defined boundaries may also be derived by Green's function techniques. The derivation, however, is more elaborate. Actually, Eq. (8.14) achieves results which go substantially beyond the classical approach:

(1) The integral equation is eatablished directly and very simply as a particular application of general thermodynamic principles.

(2) It is formulated in a very gencral way and is applicable to any thermal system and boundary conditions, no matter how intricate, provided it is linear and with time-independent parameters.

(3) The kernel $\gamma\left(x^{\prime}, x\right)$ is expressed in terms of the integrated product of associated flow fields which themselves may be evaluated by approximate variational procedures as indicated above in Section (4). In this process, conservation of energy is preserved and rough approximations may be used because of the averaging effect of the integration in evaluating expression (8.11) for $\gamma\left(x^{\prime}, x\right)$.

It will be noted that Eq. (8.14) gives directly the steady-stale temperature field if the applied external temperature $\theta_{a}$ is independent of time. Putting $\dot{\theta}=0$ we may write Eq. (8.14) as

$$
c \theta(x)=Q(x)
$$

This may also be looked upon as a consequence of reciprocity theorems for the influence coefficients relating steady temperature to heat flow, in direct analogy with Maxwell's theorem of the theory of elasticity.

An alternate formulation of Eq. (8.14) results from the introduction of an instantaneous steady-state temperature as already mentioned in reference 1 and in Sections (2), (4), and (7) above. This temperature field $\theta^{*}(x, t)$ is given in terms of $\theta_{a}(x, t)$ by

$$
c \theta^{*}(x, t)=Q(x, t)=\int_{S} \theta_{a}(x, t) \Theta_{(n)}(\xi, x) d S
$$

$\dagger$ This is done currently in theoretical physics (see, for instance, reference 9 ). We could also follow the procedure introduced by this writer in reference $4-$ i.e., replace $D$ by an operational invariant and use the variational principle directly.

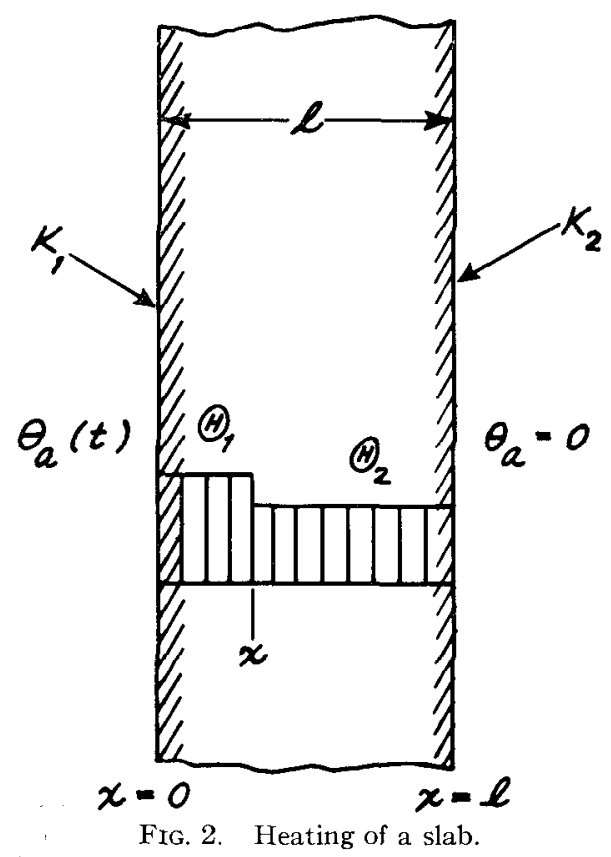

It is the temperature field which would be obtained if the instantaneous boundary temperature $\theta_{a}$ at time $t$ were maintained constant. If we now write the actual temperature as

$$
\theta(x, t)=\theta^{+}(x, t)+\theta^{*}(x, t)
$$

Eq. (8.14) becomes

$$
\begin{aligned}
c \theta^{+}(x, t)+\int \gamma\left(x^{\prime}, x\right) \dot{\theta}^{+}\left(x^{\prime}, t\right) d x^{\prime} & = \\
& \int \gamma\left(x^{\prime}, x\right) \dot{\theta}^{*}\left(x^{\prime}, t\right) d x^{\prime}
\end{aligned}
$$

The temperature $\theta^{+}$results from subjecting the same thermal system to a new thermal force equal to the right-hand side of Eq. (8.18).

This is obviously an easier equation to solve if the temperatures vary slowly. Needless to say, since Eq. (8.18) is of the same form as the original, the process of splitting the temperature into an instantaneous steady state and remainder may be repeated, leading to an iterative solution of the problem.

\section{(9) One-Dimensional Problems Formulated by} Means of Associated Flow Fields

The treatment of a one-dimensional problem by the use of associated flow fields is given here as an illustration. Its main purpose is to familiarize the reader with the nature of the method and concepts. Furthermore, it also serves to outline the approximate method of numerical solution of the integral equation.

We shall consider the particular case of a slab of thickness $l$ with constant values of $k$ and $c$. One face at $x=$ 0 has a heat-transfer coefficient $K_{1}$, and the other face at $x=l$ a heat-transfer coefficient $K_{2}$ (see Fig. 2). The outside temperature at $x=0$ is a given function of time, $\theta_{a}(t)$, while the outside temperature at $x=l$ is zero. Consider the temperature $\theta(x, t)$ at point $x$ and time $t$. The associated flow field corresponds to a concentrated heat sink in the plane of abscissa $x$ while the temperature outside of the slab is maintained at 
zero on both sides. Corresponding to Eq. (8.2) we have now the representation of the temperature field as

$$
\theta(\xi)=\int \delta(\xi-x) \theta(x) d x
$$

which is formally the same except that now $\delta(\xi-x)$ represents a one-dimensional Dirac function.

Corresponding to Eq. (8.3) the intensity of the heat sink is $c$ per unit area of the slab cross section. The associated flow field is the heat flow which would flow into the sink from both sides of the slab under a steadystate condition. The flow field is uniform on each side of point $x$ with values which are inversely proportional to the flow resistance on each side of the sink. Denoting by $\theta_{1}$ and $\theta_{2}$ the flow coming into the sink on each side, respectively, we have the two equations

$$
\begin{gathered}
\Theta_{1}+\Theta_{2}=c \\
\Theta_{1}\left[\left(1 / K_{1}\right)+(x / k)\right]=\Theta_{2}\left\{\left(1 / K_{2}\right)+[(l-x) / k]\right\}
\end{gathered}
$$

The second equation states that the temperature drop between the sink and the region outside the slab is the same on both sides of the sink. Solving for $\theta_{1}$ and $\theta_{2}$ yields the flow field associated with the temperature $\delta(\xi-x)$. It is a discontinuous function

$$
\begin{array}{ll}
\theta(\xi, x)=\theta_{1}(x) & \text { for } \xi<x \\
\theta(\xi, x)=-\theta_{2}(x) & \text { for } \xi>x
\end{array}
$$

with

$$
\begin{array}{r}
\theta_{1}(x)=\left\{c /\left[\left(1 / K_{1}\right)+\left(1 / K_{2}\right)+(l / k)\right]\right\} \times \\
\left\{\left(1 / K_{2}\right)+[(l-x) / k]\right\} \\
\theta_{2}(x)=\left\{c /\left[\left(1 / K_{1}\right)+\left(1 / K_{2}\right)+(l / k)\right]\right\} \times \\
{\left[\left(1 / K_{1}\right)+(x / k)\right]}
\end{array}
$$

The thermal force $Q$ is

$$
Q(x, t)=\theta_{a}(t) \theta(0, x)
$$

or

$$
Q(x, t)=\theta_{a}(t) \Theta_{1}(x)
$$

This is a function of the coordinate $x$ and the time $t$. The kernel $\gamma\left(x^{\prime}, x\right)$ is found by applying Eq. (8.11)

$$
\begin{aligned}
\gamma\left(x^{\prime}, x\right) & =(1 / k) \int_{0}^{l} \theta\left(\xi, x^{\prime}\right) \theta(\xi, x) d \xi+ \\
& \left(1 / K_{1}\right) \Theta_{1}\left(x^{\prime}\right) \Theta_{1}(x)+\left(1 / K_{2}\right) \Theta_{2}\left(x^{\prime}\right) \Theta_{2}(x)
\end{aligned}
$$

The integral in this expression is readily evaluated. Taking into account relations $(9.2)$ and (9.3), the result may be simplified to

$$
\left.\begin{array}{r}
\gamma\left(x^{\prime}, x\right)=c\left[(x / k)+\left(1 / K_{1}\right)\right] \theta_{1}\left(x^{\prime}\right) \\
\text { for } x<x^{\prime} \\
\gamma\left(x^{\prime}, x\right)=c\left\{[(l-x) / k]+\left(1 / K_{2}\right)\right\} \theta_{2}\left(x^{\prime}\right) \\
\text { for } x>x^{\prime}
\end{array}\right\}
$$

This may be recognized as the Grcen's function of the one-dimensional problem-i.e., it is proportional to the temperature distribution due to a source at point $x^{\prime}$. That this should be so is, of course, a consequence of the general proof in the Appendix. Obviously, in one-dimensional problems we could introduce Green's function directly instead of going through the process of calculating first the associated flow field $\theta(\xi, x)$, and the above derivation is intended only as an illustration. However, as pointed out above, the introduction of the associated flow field provides an approximate method of evaluation of Green's function for the more complex two- and three-dimensional problems.

The integral equation for the temperature is

$$
c \theta(x, t)+\int_{0}^{l} \gamma\left(x^{\prime}, x\right) \dot{\theta}\left(x^{\prime}, t\right) d x^{\prime}=\theta_{a}(t) \Theta_{1}(x)
$$

This equation is reduced to a system of linear differential equations if we divide the interval of integration into a certain number of segments, the dividing points being at abscissas $x_{i}$. We put

$$
\theta_{i}=\theta\left(x_{i}, t\right)
$$

We may then write the matrix approximation

$$
\int_{0}^{l} \gamma\left(x^{\prime}, x_{i}\right) \dot{\theta}\left(x^{\prime}, t\right) d x^{\prime}=\left[b_{i j}\right]\left[\dot{\theta}_{j}\right]
$$

If the intervals $\Delta x$ are equal and in even number the relation (9.14) may be obtained by Simpson's rule i.e., we may write

$$
\left[b_{i j}\right]=[S][\Gamma] \Delta x
$$

The matrix $[\Gamma]$ is made up of elements

$$
[\Gamma]=\left[\gamma\left(x_{j}, x_{i}\right)\right]
$$

and $[S]$ is a diagonal matrix which yields Simpson's method of integration

$$
[S]=\frac{1}{3}\left[\begin{array}{lllllll}
1 & & & & & & \\
& 4 & & & & & \\
& & 2 & & & 0 & \\
& & 4 & & & \\
& & & \cdot & & & \\
& & & \cdot & & & \\
& & & & & & \\
& & 0 & & & 4 & \\
& & & & & & 1
\end{array}\right]
$$

The integral equation (9.12) then reduces to a system of linear differential equations with a finite number of unknown temperatures $\theta_{i}$. This system is

$$
c \theta_{i}+\sum^{j} b_{i j} \dot{\theta}_{j}=\theta_{a}(t) \theta_{\mathbf{l}}\left(x_{i}\right)
$$

The system may be conveniently solved by normal coordinates because the homogeneous systems obtained by putting $\theta_{a}=0$ is in a form which can be readily used for the computation of the thermal modes by iteration starting with the mode of longest relaxation time.

It should be noted that a division of the domain in equal intervals $\Delta x$ is not the best. We may just as easily take a few smaller intervals near to the surface at which the temperature is applied, and larger intervals elsewhere. The essence of the procedure remains unchanged, but we have allowed for a more accurate representation of the temperature in the region where a 
higher resolution is required. Such a procedure coupled with the present formulation and the use of Simpson's rule should yield a very high accuracy with a relatively small number of unknowns.

As pointed out in the general treatment [see Section (8) ], the steady-state temperature distribution rcsults immediately from Eqs. (9.12) and (9.5) in explicit form without any further solution. Assuming $\theta_{\alpha}$ independent of time and putting $\dot{\theta}=0$ in Eq. (9.12) we find

$$
\theta(x)=\theta_{a} \frac{\left(1 / K_{2}\right)+[(l-x) / k]}{\left(1 / K_{1}\right)+\left(1 / K_{2}\right)+(l / k)}
$$

which, as can be verified, is the steady-state temperature under the prescribed boundary conditions. The method is also easily applied to the case of an inhomogeneous or composite slab. In this case the thermal conductivity is a function of the abscissa. Eq. (9.3) is replaced by

$$
\theta_{1}\left[\frac{1}{K_{1}}+\int_{0}^{x} \frac{d \xi}{k(\xi)}\right]=\theta_{2}\left[\frac{1}{K_{2}}+\int_{x}^{l} \frac{d \xi}{k(\xi)}\right]
$$

The factor $1 / k$ in expression (9.10) for $\gamma\left(x \cdot x^{\prime}\right)$ must remain under the integral sign. We then proceed exactly as in the case of the homogeneous slab.

\section{(10) The Associated Field for the Case of Time-} Dependent Surface Heat-Transfer Coefficients

In the above treatment of associated fields we have assumed that the surface heat-transfer coefficient $K$ may be a function of the coordinates but is independent of the time. We will now show explicitly that the concept of associated field applies also to the more general case where $K$ is also a function of time. In this case, the associatcd ficld will generally be a function of time and the corresponding differential equations will have time-dependent coefficients.

In order to show this, let us consider separately the dissipation function for the solid volume $\tau$. This dissipation function is written

$$
D_{\tau}=(1 / 2) \iiint_{\tau}(1 / k)(\partial H / \partial t)^{2} d \tau
$$

The differential equation of the thermal system may be written in terms of this dissipation function as

$$
\left(\partial V / \partial q_{i}\right)+\left(\partial D_{\tau} / \partial \dot{q}_{i}\right)=Q_{i}{ }^{\prime}
$$

where $Q_{i}{ }^{\prime}$ is now the thermal force due to the temperatures $\theta^{\prime}$ at the solid boundary-i.e.,

$$
Q_{i}{ }^{\prime}=\iint_{S} \theta^{\prime}\left[\partial H_{(n)} / \partial t\right] d S
$$

This is the same expression as Eq. (2.8), in which the external temperature $\theta_{a}$ is replaced by $\theta^{\prime}$.

If the surface heat-transfer coefficient is denoted by $K$, we may write

$$
\partial H_{(n)} / \partial t=K\left(\theta_{a}-\theta^{\prime}\right)
$$

solving for $\theta^{\prime}$ and substituting in the expression for $Q^{\prime}{ }^{\prime}$, Eqs. (10.2) are written

$$
\begin{aligned}
& \left(\partial V / \partial q_{i}\right)+\left(\partial D_{\tau} / \partial \dot{q}_{i}\right)+ \\
& \quad \iint_{S}(1 / K)\left[\partial H_{(n)} / \partial t\right]\left[\partial H_{(n)} / \partial q_{i}\right] d S=Q_{i}
\end{aligned}
$$

with

$$
Q_{i}=\iint_{S} \theta_{a}\left[\partial H_{(n)} / \partial q_{i}\right] d S
$$

These equations are obviously valid if $K$ is a function of both time and location.

Consider now a field represented as

$$
\boldsymbol{H}=\sum^{i} \Theta_{i} q_{i}+\sum^{j} \boldsymbol{F}_{j} f_{j}
$$

As before, we have split the field into two parts. One part is divergence-free-i.e.,

$$
\operatorname{div} \boldsymbol{F}_{j}=0
$$

with the corresponding ignorable coordinates written as $f_{j}$ instead of $q_{i}$. The other part yields a temperature distribution by the relations

$$
\operatorname{div} \Theta_{i}=-c \theta_{i}
$$

The temperature field is then

$$
\theta=\sum^{i} \theta_{i} q_{i}
$$

Looking at Eqs. (10.5) it is seen that the coupling terms between the coordinates $q_{i}$ and $f_{j}$ are

$$
\begin{aligned}
b_{i j}{ }^{\prime}=\iiint_{\tau}(1 / k) \boldsymbol{F}_{j} \Theta_{i} d \tau & + \\
& \iint_{S}(1 / K) F_{j n} \Theta_{i n} d S
\end{aligned}
$$

We denote by $F_{j n}$ and $\Theta_{i n}$ the components of $F_{j}$ and $\Theta_{i}$ taken positive outward. Changing from inward to outward components does not affect the result since it amounts to a double change of sign in expression (10.5). We now consider that $\boldsymbol{\Theta}_{i}$ is derived from a flow potential $\psi_{i}$

$$
\boldsymbol{\Theta}_{i}=-k \operatorname{grad} \psi_{i}
$$

This does not restrict the generality since the total flow field itself is derived from a flow potential. We substitute expression (10.11) for $\Theta_{i}$ in the volume integral of relation (10.10) and integrate by parts. Taking into account the property that $F_{j}$ is divergencefree-i.e., satisfies relation (10.7) - we find

$b_{\imath j}^{\prime}=-\iint_{S} F_{j n}\left[\psi_{i}+(k / K) \operatorname{grad}_{n} \psi_{i}\right] d S=0$

This expression vanishes if we choose the flow potentials $\psi_{i}$ such that they satisfy the condition

$$
K \psi_{i}+k \operatorname{grad}_{n} \psi_{i}=0
$$

at the boundary. On the other hand, combining Eqs. (10.8) and (10.11) we derive

$$
\operatorname{div}\left(k \operatorname{grad} \psi_{i}\right)=c \theta_{i}
$$

Hence, $\psi_{i}$ is completely determined from the temper- 
ature field $\theta_{i}$ by this last equation and the boundary condition (10.13). The flow potential $\psi_{i}$ is the steadystate temperature generated by distributed $\operatorname{sinks} c \theta_{i}$, under conditions of zero outside temperature. Although this is a steady-state problem, we must remember that the boundary condition (10.13) is variable since $K$ is a function of time. In other words, we are dealing here with a succession of instantaneous steady states. Although the distribution of heat sinks is independent of time, the flow potential $\psi_{i}$ and the associated flow fields $\Theta_{i}$ will be functions of time. This may be expressed explicitly by writing

$$
\left.\begin{array}{rl}
\theta & =\sum^{i} \theta_{i}(x, y, \xi) q_{i} \\
H & =\sum^{i} \theta_{i}(x, y, z, t) q_{i}
\end{array}\right\}
$$

With these associated flow fields the problem may be formulated by Eqs. (2.2) with only the non-ignorable coordinates $q_{i}$ appearing in the equations. The coefficients of $\dot{q}_{i}$ in the differential equations will now be functions of time.

As in the previous case discussed in Section (4), the method is applicable to the nonlinear case where the heat capacity is temperature-dependent. It is also, of course, valid for anisotropic media as can be easily verified by repeating the derivation for this case.

Of practical importance is the applicability to this case of the variational methods already outlined in Section (4) for the evaluation of the associated field. Again we do not need to calculate the flow potential $\psi_{i}$, but the vector field $\Theta_{i}$ may be derived directly from the temperature $\theta_{i}$ by a variational method. The variational formulation expresses a principle of minimum dissipation for the flow field generated by the prescribed distribution of heat sinks. We must call attention to the fact that, although the minimizing flow field is a steady-state flow, it is a continuous succession of steady states due to the time dependence of the surface heat-transfer properties.

It is, of course, not necessary to express the temperature as a linear function of $q_{i}$. The method is therefore applicable to the case where the temperature field is described by the more general function $\theta(x, y$, $\left.z, t, q_{i}\right)$. Furthermore, the surface heat-transfer coefficients may also be a function of the surface temperature. In this case, the associated flow field contains the surface temperature and the time explicitly.

\section{APPENDIX}

A direct verification of Eq. (8.14) and of the identity of $\gamma\left(x^{\prime}, x\right)$ with Green's function is obtained as follows. Let us write the following well-known identities:

$$
\begin{array}{r}
\int_{\tau} W \operatorname{div}(k \operatorname{grad} U) d \tau+\int_{\tau} k \operatorname{grad} W \operatorname{grad} U d \tau= \\
\int_{S} k W \operatorname{grad}_{n} U d S
\end{array}
$$

$$
\begin{array}{r}
\int_{\tau} U \operatorname{div}(k \operatorname{grad} W) d \tau+\int_{\tau} k \operatorname{grad} U \operatorname{grad} W d \tau= \\
\int_{S} k U \operatorname{grad}_{n} W d S
\end{array}
$$

and Green's theorem derived by writing the difference of the above identities

$$
\begin{array}{r}
\int_{\tau}[W \operatorname{div}(k \operatorname{grad} U)-U \operatorname{div}(k \operatorname{grad} W)] d \tau= \\
\int_{S} k\left(W \operatorname{grad}_{n} U-U \operatorname{grad}_{n} W\right) d S
\end{array}
$$

The left side integral is extended over the volume $\tau$ with the volume element $d \tau$ and the surface integrals over the boundary $S$ of this volume. The symbol $\operatorname{grad}_{n}$ denotes the normal component of the gradient taken positive outward.

Consider now a Green's function $g\left(x^{\prime}, x\right)$ defined by the equation

$$
\operatorname{div}(k \operatorname{grad} g)=c \delta\left(x^{\prime}-x\right)
$$

with the boundary condition

$$
K g+k \operatorname{grad}_{n} g=0
$$

Dirac's function is represented by $\delta\left(x^{\prime}-x\right)$. For abbreviation, $x$ stands for $x y z$ and $x^{\prime}$ for $x^{\prime} y^{\prime} z^{\prime}$. The differential operators act on the coordinates $x^{\prime} y^{\prime} z^{\prime}$ so that $g$ represents the temperature due to a steady heat sink of magnitude $c$ located at $x y z$. Consider also a transient thermal problem with a temperature $\theta$ satisfying the equations

$$
\operatorname{div}(k \operatorname{grad} \theta)=c(\partial \theta / \partial t)
$$

and the boundary condition

$$
K\left(\theta-\theta_{a}\right)+k \operatorname{grad}_{n} \theta=0
$$

Let us now substitute

$$
U=g, \quad W=\theta
$$

into relation (A-3). We find

$$
\begin{array}{r}
\int_{\tau}[\theta \operatorname{div}(k \operatorname{grad} g)-g \operatorname{div}(k \operatorname{grad} \theta)] d x^{\prime}= \\
\int_{S} k\left(\theta \operatorname{grad}_{n} g-g \operatorname{grad}_{n} \theta\right) d S
\end{array}
$$

The volume element $d x^{\prime} d y^{\prime} d z^{\prime}$ is written as $d x^{\prime}$. Taking into account Eqs. (A-4) and (A-6), the volume integral may be written

$$
\begin{array}{r}
\int_{\tau}[\theta \operatorname{div}(k \operatorname{grad} g)-g \operatorname{div}(k \operatorname{grad} \theta)] d x^{\prime}= \\
c \theta(x)-\int_{\tau} c g\left(x^{\prime}, x\right)\left[\partial \theta\left(x^{\prime}\right) / \partial t\right] d x^{\prime}
\end{array}
$$

Taking into account Eqs. (A-5) and (A-7), the surface integral becomes

$$
\begin{aligned}
& \int_{S} k\left(\theta \operatorname{grad}_{n} g-g \operatorname{grad}_{n} \theta\right) d S= \\
& \quad \int_{S} \theta_{a} k \operatorname{grad}_{n} g d S=Q(x, t)
\end{aligned}
$$


We have put this expression equal to $Q(x, t)$ because it coincides with the definition (8.13) of the same quantity in the text. In fact, we may write

and

$$
\left.\begin{array}{l}
\Theta=-k \operatorname{grad} g\left(x^{\prime}, x\right) \\
\Theta_{(n)}=k \operatorname{grad}_{n} g\left(x^{\prime}, x\right)
\end{array}\right\}
$$

where $\theta_{(n)}$ denotes the normal component of the boundary flow directed positively inward. Hence,

$$
Q(x, l)=\int_{S} \theta_{a}(l) \theta_{(n)}\left(x, x^{\prime}\right) d S
$$

We remember that $x^{\prime}$ plays the role of the variable in the integration and $x$ that of a parameter. Combining Eqs. (A-9), (A-10), and (A-11) we derive

$$
c \theta(x)-\int_{\tau} c g\left(x^{\prime}, x\right) \dot{\theta}\left(x^{\prime}\right) d x^{\prime}=Q(x, t)
$$

Comparing this with Eq. (8.14), we see that $-c g\left(x^{\prime}, x\right)$ plays the role of $\gamma\left(x^{\prime}, x\right)$.

That these quantities are actually identical may be verified directly by applying either one of the identities (A-1) and (A-2). Let us consider again the scalar field $g\left(x^{\prime}, x\right)$ defined by (A-4) and (A-5) replacing $x^{\prime}$ by the coordinate $\xi$ (an abbreviation for $\xi n \zeta$ ). In this case, $\xi$ plays the role of the running coordinates and $x$ that of parameters representing the location of the source. Let us consider two such functions, $g(\xi, x)$ and $g\left(\xi, x^{\prime}\right)$, and put

$$
W=g(\xi, x), \quad U=q\left(\xi, x^{\prime}\right)
$$

Applying identity (A-1) we find

$\int_{\tau} g(\xi, x) \operatorname{div}\left[k \operatorname{grad} g\left(\xi, x^{\prime}\right)\right] d \tau+$

$$
\begin{array}{r}
\int_{\tau} k \operatorname{grad} g(\xi, x) \operatorname{grad} g\left(\xi, x^{\prime}\right) d \tau= \\
\int_{S} k g(x, \xi) \operatorname{grad}_{n} g\left(\xi, x^{\prime}\right) d S
\end{array}
$$

We write

$$
\left.\begin{array}{r}
\boldsymbol{\Theta}(\xi, x)=-k \operatorname{grad} g(\xi, x) \\
\Theta\left(\xi, x^{\prime}\right)=-k \operatorname{grad} g\left(\xi, x^{\prime}\right)
\end{array}\right\}
$$

Furthermore we utilize Eqs. (A-4) and (A-5), applying the first one to $g\left(\xi, x^{\prime}\right)$ and the second one to $g(\xi, x)$.

$$
\left.\begin{array}{l}
\operatorname{div}\left[k \operatorname{grad} g\left(\xi, x^{\prime}\right)\right]=c \delta\left(\xi, x^{\prime}\right) \\
K g(\xi, x)+k \operatorname{grad}_{n} g(\xi, x)=0
\end{array}\right\}
$$

Introducing these expressions in the volume and surface integrals of Eq. (A-16), we derive

$$
\begin{array}{r}
c g\left(x^{\prime}, x\right)=-\int_{\tau} \frac{1}{k} \Theta_{n}\left(x^{\prime}, \xi\right) \Theta_{n}(x, \xi) d \tau- \\
\int_{S} \frac{1}{K} \Theta_{n}\left(x^{\prime}, \xi\right) \Theta_{n}(x, \xi) d S
\end{array}
$$

Comparing this with expression (8.11), we conclude

$$
\gamma\left(x^{\prime}, x\right)=-\operatorname{cg}\left(x^{\prime}, x\right)
$$

thereby establishing the identity of this function with the classical Green's function type defined by Eqs. (A-4) and (A-5).

\section{REFERENCES}

1 Biot, M. A., New Methods in Heat Flow Analysis with Application to Flight Structures, Cornell Aeronautical Laboratory Report No. SA-987-S-3, May, 1956; see also, Journal of the Aeronautical Sciences, Vol. 24, No. 12, pp. 857-873, December, 1957.

${ }^{2}$ Biot, M. A., Variational Methods in Irreversible Thermodynamics with Application to Viscoelasticity, The Physical Review, Vol. 97, No. 6, pp. 1463-1479, March 15, 1955.

${ }^{3}$ Biot, M. A., Theory of Stress-Strain Relations in Anisotropic Viscoelasticity and Relaxation Phenomena, J. Appl. Phys., Vol. 25, No. 11, pp. 1385-1391, November, 1954.

${ }^{4}$ Biot, M. A., Thermoelasticity and Irreversible Thermodynamics, J. Appl. Phys., Vol. 27, No. 3, pp. 240-253, March, 1956.

- Biot, M. A., Linear Thermodynamics and the Mechanics of Solids, Cornell Aeronautical Laboratory Report April, 1958; Proceedings, Third U.S. National Congress of Applied Mechanics, ASME, June, 1958.

- Rosen, P., On Variational Principles for Irreversible Processes, J. Chem. Phys., Vol. 21, No. 7, pp. 1220-1221, July, 1953.

7 Chambers, L. C., A Variational Principle for the Conduction of Heat, Quart. J. Mech. \& Appl. Math., Vol. 9, Part 2, pp. 234235, June, 1956.

${ }^{8}$ McLachlan, N. W., Bessel Functions for Engineers, University Press, New York, 1934.

${ }^{9}$ Goldstein, H., Classical Mechanics, Chapter XI, AddisonWesley Press, Inc., Reading, Mass., 1951.

${ }^{10}$ Carslaw, H. S., and Jaeger, J. C., Conduction of Heat in Solids, Oxford, Clarendon Press, London, England, 1948. 